\title{
Clinical significance of GLUT-1 expression in patients with esophageal cancer treated with concurrent chemoradiotherapy
}

\author{
ITARU CHIBA $^{1 *}$, KAZUHIKO OGAWA ${ }^{1 *}$, TAKAMITSU MORIOKA ${ }^{2}$, \\ HIDEAKI SHIMOJI $^{3}$, NAO SUNAGAWA ${ }^{2}$, SHIRO IRAHA $^{1}$, \\ TADASHI NISHIMAKI $^{3}$, NAOMI YOSHIMI ${ }^{2}$ and SADAYUKI MURAYAMA $^{1}$ \\ Departments of ${ }^{1}$ Radiology, ${ }^{2}$ Pathology and ${ }^{3}$ Surgery, University of the Ryukyus, Okinawa, Japan
}

Received July 19, 2010; Accepted October 15, 2010

DOI: $10.3892 /$ ol.2010.199

\begin{abstract}
This study aimed to investigate whether glucose transporter-1 (GLUT-1) expression in a pretreatment esophageal cancer biopsy was predictive of clinical outcomes in patients with esophageal cancer undergoing concurrent chemoradiotherapy (CRT). A total of 25 patients with esophageal cancer treated with concurrent CRT were reviewed. Radiotherapy was administered up to total doses of 40-66.6 Gy (median 66.6 Gy) with a single fraction of 1.8-2 Gy. Regarding chemotherapy, cisplatin $\left(80 \mathrm{mg} / \mathrm{m}^{2}\right.$ on day 1$)$ and 5 -fluorouracil $\left(800 \mathrm{mg} / \mathrm{m}^{2}\right.$ on days 2-6) were used concurrently with radiotherapy, every 3-4 weeks for a total of 1-2 courses. Tissue samples from esophageal carcinoma were obtained from the 25 patients by biopsy prior to concurrent CRT, and a semiquantitative analysis of GLUT-1 expression was performed using immunohistochemical staining. High GLUT-1 expression was observed in 7 of 25 (28\%) patients, and GLUT-1 expression was significantly correlated with clinical $\mathrm{T}$ stage $(\mathrm{p}=0.0454)$, clinical $\mathrm{N}$ stage $(\mathrm{p}=0.0324)$ and initial response to CRT $(p=0.0185)$. Patients with a high GLUT-1 expression had significantly poorer local control (LC) (5-year LC 28.6\%) than those with a low expression (5-year LC 73.4\%, p<005). Multivariate analysis revealed that GLUT-1 and the number of chemotherapy courses were independent prognostic factors for LC. Patients with a high GLUT-1 expression had significantly lower recurrence-free survival (RFS) compared to those with a low GLUT-1 expression $(\mathrm{p}=0.0405)$. Multivariate analysis revealed that GLUT-1, the number of chemotherapy courses and clinical $\mathrm{M}$ stage were independent prognostic factors for RFS. GLUT-1 expression was significantly correlated with clinical T stage, clinical $\mathrm{N}$ stage and initial response to concur-
\end{abstract}

Correspondence to: Dr Kazuhiko Ogawa, Department of Radiology, University of the Ryukyus School of Medicine, 207 Uehara, Nishihara-cho, Okinawa 903-0215, Japan

E-mail: kogawa@med.u-ryukyu.ac.jp

*Contributed equally

Key words: glucose-transporter-1, esophageal neoplasm, radiotherapy, chemotherapy rent CRT, and was predictive of LC and RFS for patients with esophageal cancer treated with concurrent CRT.

\section{Introduction}

Esophageal cancer is one of the most difficult malignancies to cure, and the prognosis for these patients is poor (1-3). Although surgery is the main curative treatment for these tumors, chemoradiotherapy (CRT) was identified as a viable option for the treatment of esophageal carcinoma. The Radiation Therapy Oncology Group (RTOG) 8501 trial showed that CRT is superior to radiotherapy alone as primary treatment (4). However, a number of studies demonstrated that the patterns of failure observed following definitive CRT showed that locoregional failure is frequent, with approximately $50 \%$ of patients experiencing local failure $(4,5)$. The identification of predictive markers for the response to CRT may improve patient selection and allow for response modifications such as more intensive treatments for poor responders.

Glucose-transporter-1 (GLUT-1) is a membrane receptor that mediates the passive export of glucose across plasma membranes and its expression increases in hypoxic conditions due to reduced oxidative phosphorylation and hypoxia inducible factor-1 (HIF-1) induction (6). GLUT-1 facilitates the metabolic adaptation of cells to hypoxia and is essential for survival and proliferation of glycolytic metabolism $(7,8)$. Therefore, GLUT-1 expression may be a suitable marker of hypoxia and glucose metabolism, measured simply and cost-effectively as part of the routine histological assessment of tumors $(9,10)$. GLUT-1 has been immunohistochemically detected in a variety of malignant tissues, including tumors of the breast, thyroid, head and neck, bladder and lung (11-15). In all cases, the tumor expression was increased as compared to that of the corresponding normal tissues. The over-expression of GLUT-1 in tumors was reported to be a marker for poor prognosis in colorectal, ovarian and non-small cell lung cancers (16-18). In esophageal cancer, a number of studies showed that GLUT-1 over-expression was associated with tumor aggressiveness and poor prognosis in patients with esophageal cancer treated with surgical resection $(19,20)$. However, little information is available on the clinical significance of GLUT-1 expression in patients with esophageal cancer treated with concurrent CRT. 
Table I. GLUT-1 expression and clinicopathological characteristics in 25 patients with esophageal cancer treated with concurrent chemoradiotherapy.

GLUT-1 expression

\begin{tabular}{|c|c|c|c|c|}
\hline \multirow{2}{*}{ Variable } & \multirow[b]{2}{*}{ No. of patients } & & \multirow[b]{2}{*}{ P-value } \\
\hline & & Negative & Positive & \\
\hline \multicolumn{5}{|l|}{ Gender } \\
\hline Male & 24 & 18 & 6 & 0.1017 \\
\hline Female & 1 & 0 & 1 & \\
\hline \multicolumn{5}{|l|}{ Age (years) } \\
\hline$<60$ & 8 & 4 & 4 & 0.1663 \\
\hline$\geq 60$ & 14 & 11 & 3 & \\
\hline \multicolumn{5}{|c|}{ Clinical T stage (UICC 2002) } \\
\hline $\mathrm{T} 1-3$ & 15 & 13 & 2 & 0.0454 \\
\hline $\mathrm{T} 4$ & 10 & 5 & 5 & \\
\hline \multicolumn{5}{|c|}{ Clinical N stage (UICC 2002) } \\
\hline No & 8 & 8 & 0 & 0.0324 \\
\hline N1 & 17 & 10 & 7 & \\
\hline \multicolumn{5}{|c|}{ Clinical M stage (UICC 2002) } \\
\hline M0 & 20 & 16 & 4 & 0.0747 \\
\hline M1 & 5 & 2 & 3 & \\
\hline \multicolumn{5}{|l|}{ KPS (\%) } \\
\hline $100-70$ & 23 & 17 & 6 & 0.4701 \\
\hline$\leq 60$ & 2 & 1 & 1 & \\
\hline \multicolumn{5}{|l|}{ Tumor site } \\
\hline $\mathrm{Ce}$ or Ut & 12 & 8 & 4 & 0.5682 \\
\hline Mt or Lt & 13 & 10 & 3 & \\
\hline \multicolumn{5}{|c|}{ Total radiation dose } \\
\hline$<60$ Gy & 3 & 2 & 1 & 0.8264 \\
\hline$\geq 60 \mathrm{~Gy}$ & 22 & 16 & 6 & \\
\hline \multicolumn{5}{|c|}{ No. of chemotherapy courses } \\
\hline 1 & 7 & 4 & 3 & 0.3021 \\
\hline 2 & 18 & 14 & 4 & \\
\hline
\end{tabular}

UICC, International Union Against Cancer; KPS, Karnofsky performance status; Ce, cervical esophagus; Ut, upper thoracic; Mt, middle thoracic; Lt, lower thoracic.

In the current study, GLUT-1 expression was retrospectively semiquantitated. Moreover, whether or not these levels were associated with clinicopathological characteristics and the clinical outcomes in patients with esophageal cancer treated with concurrent CRT was investigated.

\section{Materials and methods}

Patients and sample collection. Between 1997 and 2002, 37 patients with esophageal cancer were treated with concurrent CRT at the University of the Ryukyus Hospital, Japan. The study comprised primary esophageal carcinoma specimens from pretreatment biopsies obtained from 25 patients. The disease characteristics of the 25 patients, including tumor stage and tumor sites, were not significantly different compared to those of the 12 patients from which carcinoma specimens were not obtained. The histopathological diagnosis of the 25 patients was squamous cell carcinoma. No patients received chemotherapy or radiotherapy prior to the biopsy. The carcinoma specimens were obtained from the tumor edge, thereby avoiding the necrotic center. The specimens were immediately fixed in $10 \%$ buffered formalin.

The patient characteristics of the 25 patients are shown in Table I. Of the 25 patients, 1 was female and 24 were male, and the ages ranged from 45-78 years (median 62). The study was performed according to the guidelines approved by the institutional review board of our institution, and written informed consent was obtained from the 25 patients.

Concurrent chemoradiotherapy. External beam radiotherapy (EBRT) was administered with megavoltage equipment of photon energies $\geq 4 \mathrm{MeV}$. The total doses of EBRT ranged 
from 40-66.6 Gy with a single fraction of 1.8-2 Gy 5 days per week. The median total dose of the 25 patients was $66.6 \mathrm{~Gy}$, and 22 of 25 patients $(88.0 \%)$ were treated with a total dose of $\geq 60 \mathrm{~Gy}$. The treatment field of EBRT consisted of a localized field in 4 of 25 patients $(16 \%)$, and the primary tumor plus regional lymph nodes in the remaining $21(84 \%)$. In the majority of patients, computed tomography (CT)-based treatment planning and conformal radiotherapy were used. Initially, anterior-posterior opposed fields were employed at doses of 32.4-40 Gy, and a booster dose of 14-34.2 Gy was administered, using bilateral oblique or multiple fields. The clinical target volume for the primary tumor was defined as the gross tumor volume plus $3 \mathrm{~cm}$ craniocaudally. The planning target volumes for the primary tumor and the metastatic lymph nodes were determined with 1 to $1.5 \mathrm{~cm}$ margins to compensate for set-up variations and internal organ motion. Lung heterogeneity corrections were not used.

Chemotherapy was administered concurrently with radiotherapy. One course of chemotherapy consisted of cisplatin $\left(80 \mathrm{mg} / \mathrm{m}^{2}\right.$ on day 1) and 5-fluorouracil $\left(800 \mathrm{mg} / \mathrm{m}^{2}\right.$ on days 2-6), with 3- to 4-week intervals for a total of 1-2 courses. The patients received antiemetics with granisetron and metoclopramide prior to chemotherapy administration.

Immunohistochemical staining for glucose-transporter-1 and evaluation of staining. Formalin-fixed, paraffinembedded tumor sections were dewaxed in xylene and dehydrated using a series of ethanol solutions of increasing dilution. Staining for GLUT-1 was then perfromed using the Envision Dual link. This protocol uses a 3,3'diaminobenzidine substrate system that enabled visualization of the GLUT-1 protein as a brown stain. For GLUT-1 staining, a $1 / 100(10 \mathrm{mg} / \mathrm{ml}$ protein) concentration of affinity-pure rabbit antihuman GLUT-1 (Chemicon Europe) was utilized. An incubation time was performed overnight at $4^{\circ} \mathrm{C}$ for the primary antibody steps, whereas an incubation time of 30 min at room temperature was selected for each secondary antibody. The negative controls were prepared by omitting the primary antibodies. Following staining, sections were rinsed with water, counterstained with Gill's hematoxylin and coverslipped using an aqueous mountant.

Two independent pathologists blinded to the clinicopathological data performed the scoring of the immunohistochemical staining. The percentage of positive tumor cells was semiquantitatively determined by assessing the whole biopsy specimen, and the mean percentage of positive tumor cells was calculated by the two pathologists. In the current study, each sample was characterized as either 1, low (0-30\%); or 2 , high $(31-100 \%)$.

Statistical analysis. The median follow-up of 9 surviving patients was 57.8 months (range 2.8-107.7). In the current study, the initial response of the primary tumor was evaluated using the criteria of the Japanese Society for Esophageal Disease, which were based on findings from esophagograms and esophagoscopies (21). In brief, complete response (CR) was defined as the complete disappearance of tumor and no appearance of new lesions at least 4 weeks after treatment. Partial response (PR) was defined as a $>50 \%$ reduction in the product of the perpendicular diameters of the tumor and no appearance of new lesions at least 4 weeks after treatment. Progressive disease (PD) was defined as a $>25 \%$ increase in the product of the perpendicular diameters of the tumor or any new tumor. Any further conditions were defined as no change (NC). Disease recurrence was defined as recurrence or progression of the initial disease or the occurrence of new disease following CRT detected by a CT scan and/or esophagoscopy, which were performed every 3-4 months for 2 years and then twice a year. Overall survival (OS), recurrence-free survival (RFS) and local control (LC) rates were calculated actuarially according to the Kaplan-Meier method (22), and were measured from the first day of CRT. Differences between the groups were estimated using the Chi-square and generalized Wilcoxon tests (23). Multivariate analysis was performed using the Cox regression model (24). $\mathrm{P}=0.05$ was considered to be statistically significant. Statistical analysis was performed with the SPSS software package (version 6.1; SPSS, Inc., Chicago, IL, USA).

\section{Results}

Representative examples of high or low GLUT-1 expression by immunohistochemical analysis are shown in Fig. 1. In the current study, a high GLUT-1 expression was observed in 7 of $25(28 \%)$ patients, and the patients were divided into low and high GLUT-1 expression groups. Table I shows the clinical data and GLUT-1 expression in the tumor biopsies of the 25 patients. The proportion of patients with T4 tumors was significantly higher $(\mathrm{p}=0.0454)$ in the high GLUT-1 expression group than that of patients with low GLUT-1 expression. Moreover, the proportion of patients with N1 tumor was significantly higher $(\mathrm{p}=0.0324)$ in the high GLUT-1 expression group than that of patients with low GLUT-1 expression. Other characteristics, such as age and gender, did not correlate with the GLUT-1 expression.

Table II shows GLUT-1 expression and the initial response in 25 patients. A total of 12 of 13 patients (92.3\%) exhibited a CR response in the low GLUT-1 expression group, while 6 of 12 patients $(50.0 \%)$ exhibited a CR response in the high GLUT-1 expression group. Significant differences were noted between the low and high GLUT-1 expression groups regarding the initial response to concurrent CRT $(\mathrm{p}=0.0185)$.

At the time of this study, 16 patients $(91.0 \%)$ had disease recurrences (local, 6 patients; regional lymph nodes, 2; distant metastasis, including bone or lung, 4; and multiple sites, 4 patients). Of the 16 patients with multiple recurrences, 2 patients had simultaneous local recurrence. Local recurrence occurred in 8 patients $(32.0 \%)$ in total. The 5-year actuarial LC rate in the 25 patients was $61.9 \%$. Fig. 2 shows the LC curves according to the GLUT-1 expression. Patients with high GLUT-1 expression had a significantly poorer LC (5-year LC; 28.6\%) than those with low GLUT-1 expression (5-year LC; 73.4\%; p<005). The univariate analysis showed that GLUT-1 expression, the number of chemotherapy courses, total radiation dose and clinical $M$ stage had a significant impact on LC (Table III), while the multivariate analysis showed that GLUT-1 expression and the number of chemotherapy courses were independent prognostic factors for LC (Table IV). 
A

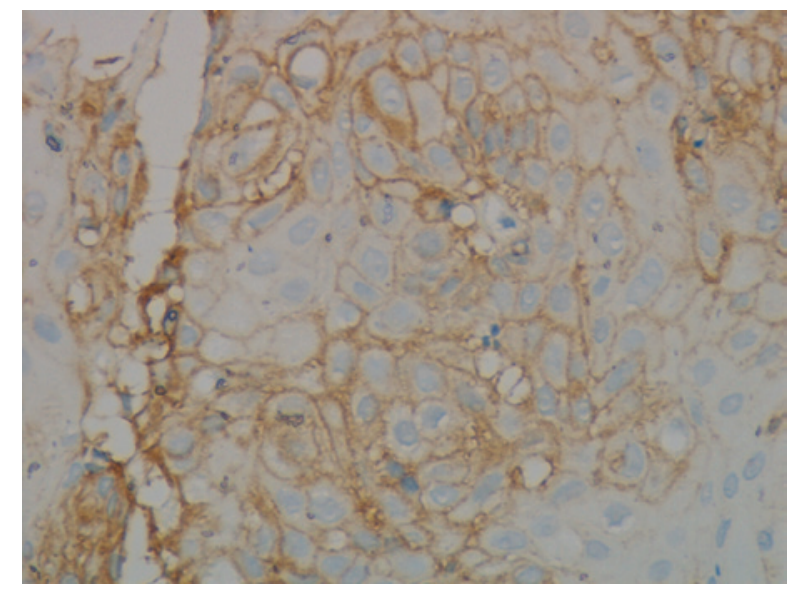

B

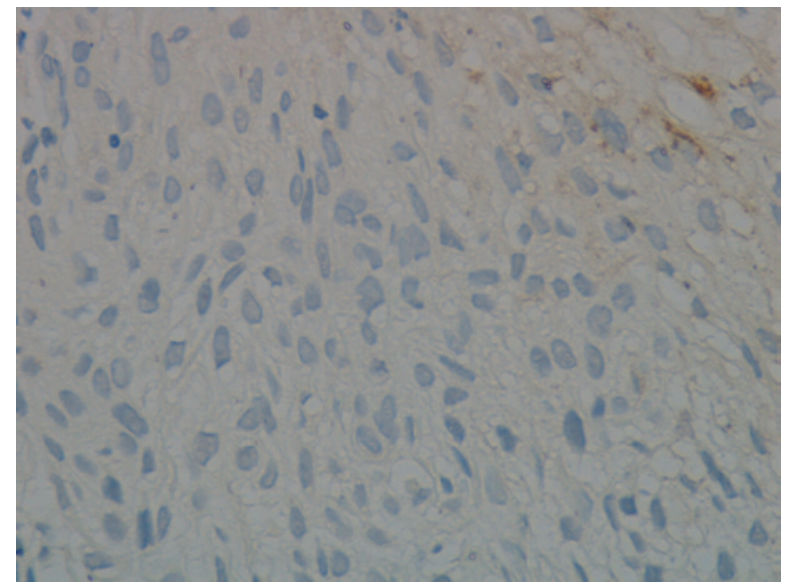

Figure 1. Representative example of GLUT-1 expression in esophageal carcinoma. Immunohistochemical staining with anti-GLUT-1 antibody in esophageal carcinoma cells. (A) High and (B) low GLUT-1 expression.

Table II. GLUT-1 expression and initial response in 25 patients with esophageal cancer treated with concurrent chemoradiotherapy.

\begin{tabular}{lcrcr}
\hline \multirow{2}{*}{$\begin{array}{l}\text { Initial } \\
\text { response }\end{array}$} & $\begin{array}{c}\text { No. of } \\
\text { patients }\end{array}$ & \multicolumn{2}{c}{ GLUT-1 expression } & \\
\cline { 3 - 4 } & & Low (\%) & High (\%) & P-value \\
\hline CR & 13 & $12(92.3)$ & $1(7.7)$ & 0.0185 \\
PR or NC & 12 & $6(50.0)$ & $6(50.0)$ & \\
\hline
\end{tabular}

GLUT-1, glucose transporter 1; CR, complete response; PR, partial response; $\mathrm{NC}$, no change.

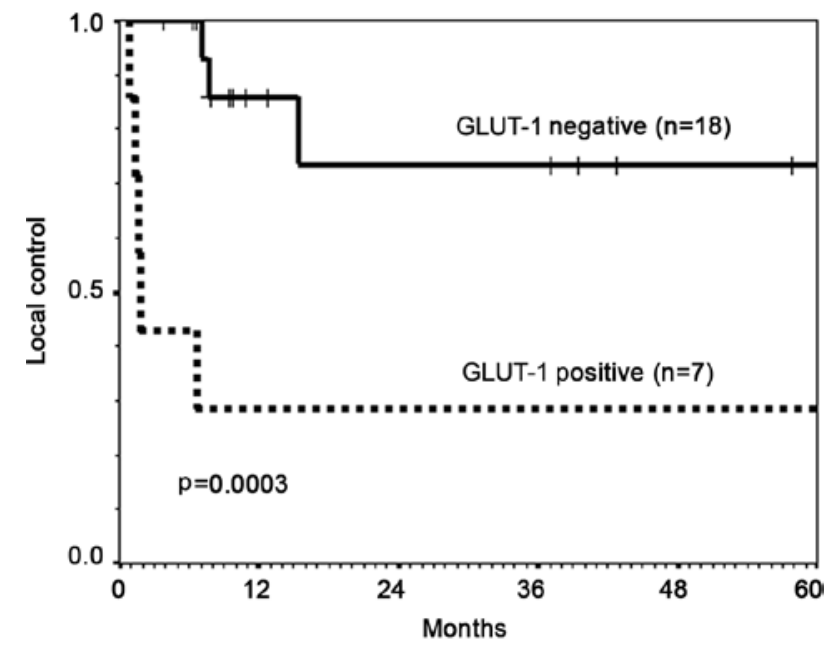

Figure 2. Local control curves according to the GLUT-1 expression in esophageal carcinoma patients treated with concurrent chemoradiotherapy.

Of the 25 patients, 16 (64.0\%) succumbed to the disease during this study. Of the 16 patients, 13 succumbed to esophageal carcinoma and the remaining 3 patients succumbed

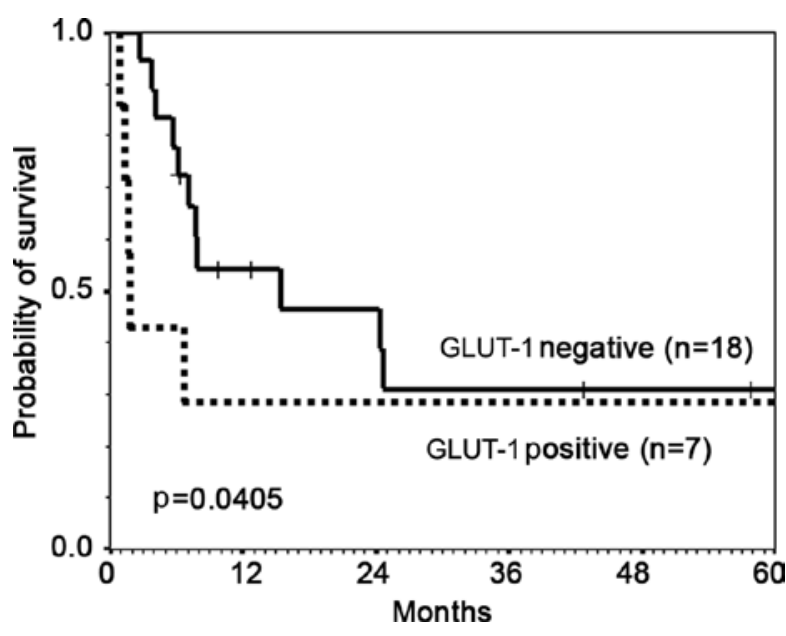

Figure 3. Disease-free survival curves according to the GLUT-1 expression in esophageal carcinoma patients treated with concurrent chemoradiotherapy.

without any sign of clinical recurrence (1, radiation pneumonitis; 1, pneumonia; and 1, unknown causes). The 5-year actuarial OS and RFS rate for the 25 patients was 27.3 and $31.3 \%$, respectively. Fig. 3 shows the RFS curves according to GLUT-1 expression. Patients with a high GLUT-1 expression had a significantly lower RFS compared to those with a low GLUT-1 expression ( $\mathrm{p}=0.0405)$. The univariate analysis showed that GLUT-1 expression, the number of chemotherapy courses and clinical M stage had a significant impact on OS, while the multivariate analysis, the three factors proved to be significant prognostic factors for RFS (Tables V and VI).

Late complications of NCI-CTC Grade 4-5 were observed in 2 patients (4.0\%). One patient suffered grade 4 pericardical effusion requiring pericardial puncture 58 months after the completion of CRT. The remaining patient suffered grade 5 radiation pneumonitis and succumbed to radiation pneumonitis 4 months after CRT. The 2 patients were treated with a total dose of 66.6 Gy radiotherapy and 2 courses of chemotherapies. 
Table III. Univariate analysis of various potential prognostic factors for LC in patients with esophageal cancer treated with concurrent chemoradiotherapy.

Univariate analysis

No. of patients

LC, 5 -year rate $(\%)$

P-value

Gender

Male

24

Female

$\geq 60$ or more

T4

N1

M1

\section{Mt or Lt}

59.7

100

60.0

0.5065 thoracic; Lt, lower thoracic.

\section{Discussion}

The current study showed that GLUT-1 expression is significantly correlated with clinical $\mathrm{T}$ and $\mathrm{N}$ stages in patients with esophageal carcinoma. The percentage of patients with T4 tumor was significantly higher in the high GLUT-1 expression group than that of patients with low GLUT-1 expression. Moreover, the percentage of patients with N1 tumor was significantly higher in the high GLUT-1 expression group than that of patients with a low GLUT-1 expression. A number of studies found an association between GLUT-1 expression and tumor stage $(19,20)$. Tohma et al found that pathological T3-4 esopha- geal cancers showed higher percentages of GLUT-1 strong positive cells than T1-2 cancers, and lesions exhibiting lymph node metastasis showed higher percentages of GLUT-1 strong positive cells than lesions without lymph node metastasis (19). Kato et al showed that significant correlations between GLUT-1 expression and tumor stage were observed in patients with esophageal squamous cell carcinoma (20). The results of these authors, together with our results, proved that GLUT-1 expression is correlated with tumor aggressiveness in patients with esophageal carcinoma. GLUT-1 may be linked to invasiveness by supporting glycosis, which enhances tumor cell viability and provides energy for cell division and tumor growth (25). 
Table IV. Multivariate analysis of various potential prognostic factors for LC in patients with esophageal cancer treated with concurrent chemoradiotherapy.

\begin{tabular}{lccr}
\hline & & Multivariate analysis & \\
\cline { 3 - 3 } & & RR (95\% CI) & P-value \\
\hline GLUT-1 & (Low vs. high) & $0.047(0.005-0.434)$ & 0.0070 \\
Chemotherapy course & (1 course vs. 2 courses) & $29.598(2.872-304.993)$ & 0.0040 \\
Clinical M stage & (M0 vs. M1) & $0.260(0.001-45.361)$ & 0.6090 \\
Total radiation dose & $(<60$ Gy vs. $\geq 60$ Gy) & $0.683(0.000-3409.927)$ & 0.9300 \\
\hline
\end{tabular}

LC, local control; RR, relative ratio; CI, confidence intervals.

Table V. Univariate analysis of various potential prognostic factors for RFS in patients with esophageal cancer treated with concurrent chemoradiotherapy.

Univariate analysis

No. of patients

RFS, 5-year rate (\%)

P-value

Gender

Male

24

27.9

0.3125

Female

1

100

Age (years)

$<60$

8

25.0

0.3782

$\geq 60$

14

34.2

Clinical T stage (UICC 2002)

T1-3

$\mathrm{T} 4$

40.0

Clinical N stage (UICC 2002)

N0

60.0

0.0566

N1

Clinical M stage (UICC 2002)

M0

M1

KPS (\%)

100-70

$\leq 60$

Tumor site

$\mathrm{Ce}$ or Ut

Mt or Lt

Total radiation dose

$<60 \mathrm{~Gy}$

$\geq 60$ Gy

No. of chemotherapy courses

1

GLUT-1 expression 
Table VI. Multivariate analysis of various potential prognostic factors for DFS in patients with esophageal cancer treated with concurrent chemoradiotherapy.

\begin{tabular}{lccr}
\hline & & Multivariate analysis & \\
& & RR (95\% CI) & P-value \\
\hline GLUT-1 & (Low vs. high) & $0.113(0.019-0.668)$ & 0.0160 \\
Chemotherapy course & (1 course vs. 2 courses) & $6.614(1.473-25.797)$ & 0.0130 \\
Clinical M stage & (M0 vs. M1) & $0.065(0.008-0.513)$ & 0.0090 \\
Total radiation dose & (<60 Gy vs. $\geq 60$ Gy) & $2.580(0.332-20.005)$ & 0.3650 \\
Clinical N stage & (N0 vs. N1) & $4.474(0.958-20.892)$ & 0.0570 \\
\hline
\end{tabular}

DFS, disease-free survival; RR, relative ratio; CI, confidence intervals.

The current study showed that GLUT-1 expression was significantly correlated with initial response to concurrent CRT, and patients with a high GLUT-1 expression had a significantly poorer LC (5-year LC, 28.6\%) than those with a low expression (5-year LC, 73.4\%; $\mathrm{p}<005)$. Multivariate analysis revealed that GLUT-1 and the number of chemotherapy courses were independent prognostic factors for LC. The relationship between GLUT-1 expression and the reduction in response to radiotherapy and chemotherapy is explained by the fact that GLUT-1 expression is a marker of cellular adaptive responses to hypoxia (26-28). The biological effect of radiotherapy was reported to have increased approximately 3 -fold when irradiation was performed under well-oxygenated conditions compared to anoxic ones (29). Regarding rectal cancers, Brophy et al found that GLUT-1 expression was significantly associated with a reduced response to CRT (26). These authors showed that GLUT-1 expression appears to be a surrogate for tumor response to CRT in patients with esophageal carcinoma.

Regarding RFS, patients with a high GLUT-1 expression had significantly lower RFS compared to those with a low GLUT-1 expression $(\mathrm{p}=0.0405)$. The multivariate analysis showed that GLUT-1, the number of chemotherapy courses and clinical $\mathrm{M}$ stage were independent prognostic factors for RFS. Previous studies found that the GLUT-1 over-expression in esophageal carcinoma is a risk factor for mortality $(19,20)$ The results of these authors, together with our results, suggest that GLUT-1 expression is predictive of clinical outcomes in patients with esophageal carcinoma treated with CRT.

It is well established that hypoxic conditions due to reduced oxidative phosphorylation, which may be a consequence of the increased proliferation observed in tumors, enhances GLUT-1 expression (6,30). GLUT-1 is considered to enhance glycolytic metabolism which has been correlated with tumor proliferative activity (8). Therefore, GLUT-1 expression appears to be a surrogate marker for not only hypoxia but also the metabolic activity of malignant tumors (31). Since the presence of hypoxia causes treatment resistance by radiotherapy and chemotherapy, and the high metabolic activity causes a higher proliferative activity in tumors, a high GLUT-1 expression may result in poor treatment outcome in patients with malignant tumors.
The increase in glucose transport noted in malignant tumors has also been detected using 18-fluorodeoxyglucose positron emission tomography (FDG-PET), which can quantify tumor glucose consumption $(32,33)$. Regarding esophageal carcinoma, Taylor et al found that in patients undergoing surgical resection, increasing standardized uptake values (SUVmax) correlated with an increased GLUT-1 expression (34). GLUT-1 activity enhanced glycolytic metabolism which has been correlated with tumor proliferative activity (8). Westerterp et al showed that in patients with esophageal carcinoma, a significant correlation was found between FDG uptake and GLUT-1 expression (35). Kato et al found that GLUT-1 expression was related to FDG uptake and assessment of both FDG uptake and GLUT-1 expression may be useful for providing prognostic data in patients with esophageal carcinoma (36). However, GLUT-1 expression is obtained from superficial biopsy tissues and may not be representative of the tumor as a whole. Further studies are therefore required to investigate the correlations of GLUT-1 and FDG-PET in patients with esophageal carcinoma.

In conclusion, our results showed that GLUT-1 expression is significantly correlated with initial $\mathrm{T}$ and $\mathrm{N}$ stages, and response to concurrent CRT. Moreover, GLUT-1 is predictive of LC and DFS for patients with esophageal carcinoma treated with concurrent CRT. These findings suggest a role for GLUT-1 as a new prognostic biomarker for patients with esophageal cancer treated with CRT, and may allow for the selection of patients most likely to benefit from more intensive treatments. Furthermore, understanding the biological role of GLUT-1 may allow for response modification by targeting specific pathways. However, this study is a retrospective study with a relatively limited number of patients. Therefore further prospective studies are required to confirm our results.

\section{References}

1. Roth JA, Putnum JB Jr, Rich TA and Forastiere AA: Cancer of the esophagus. In: Cancer: Principles And Practice Of Oncology. 5th edition. Devita VT Jr, Hellman S and Rosenberg SA (eds). Lippincott-Raven, PA, pp980-1021, 1997.

2. Earlam R and Cunha-Melo JR: Oesophageal squamous cell carcinoma: I. A critical review of surgery. Br J Surg 67: 381-390, 1980. 
3. Earlam R and Cunha-Melo JR: Oesophogeal squamous cell carcinoms: II. A critical view of radiotherapy. Br J Surg 67: $457-461,1980$

4. Cooper JS, Guo MD, Herskovic A, et al: Chemoradiotherapy of locally advanced esophageal cancer: long-term follow-up of a prospective randomized trial (RTOG 85-01). Radiation Therapy Oncology Group. JAMA 281: 1623-1627, 1999.

5. Minsky BD, Pajak TF, Ginsberg RJ, et al: INT 0123 (Radiation Therapy Oncology Group 94-05) phase III trial of combinedmodality therapy for esophageal cancer: high-dose versus standard-dose radiation therapy. J Clin Oncol 20: 1167-1174, 2002.

6. Behrooz A and Ismail-Beigi F: Dual control of glut1 glucose transporter gene expression by hypoxia and by inhibition of oxidative phosphorylation. J Biol Chem 272: 5555-5562, 1997.

7. Lin Z, Weinberg JM, Malhotra R, Merritt SE, Holzman LB and Brosius FC III: GLUT-1 reduces hypoxia-induced apoptosis and JNK pathway activation. Am J Physiol Endocrinol Metab 278: 958-966, 2000.

8. Pessin JE and Bell GI: Mammalian facilitative glucose transporter family: structure and molecular regulation. Annu Rev Physiol 54: 911-930, 1992.

9. Airley R, Loncaster J, Davidson S, Bromley M, Roberts S, Patterson A, Hunter R, Stratford I and West C: Glucose transporter glut-1 expression correlates with tumor hypoxia and predicts metastasis-free survival in advanced carcinoma of the cervix. Clin Cancer Res 7: 928-934, 2001.

10. Airley RE, Loncaster J, Raleigh JA, et al: GLUT-1 and CAIX as intrinsic markers of hypoxia in carcinoma of the cervix: relationship to pimonidazole binding. Int J Cancer 104: 85-91, 2003.

11. Brown RS and Wahl RL: Overexpression of Glut-1 glucose transporter in human breast cancer. Cancer 72: 2979-2985, 1993.

12. Haber RS, Weiser RL, Pritsker A, Reder I and Burstein DE: Glut-1 glucose transporter in benign and malignant thyroid nodules. Thyroid 7: 363-367, 1997.

13. Mellanen P, Minn H, Grenman R and Harkonen P: Expression of glucose transporters in head and neck tumors. Int J Cancer 56 622-629, 1994

14. Chang S, Lee S, Lee C, Kim JI and Kim Y: Expression of the human erythrocyte glucose transporter in transitional cell carcinoma of the bladder. Urology 55: 448-452, 2000.

15. Kurata $T$, Oguri $T$, Isobe $T$, Ishioka $S$ and Yamakido $M$ : Differential expression of facilitative glucose transporter (GLUT) genes in primary lung cancers and their liver metastases. Jpn J Cancer Res 90: 1238-1243, 1999.

16. Haber RS, Rathan A, Weiser KR, et al: GLUT-1 glucose transporter expression in colorectal carcinoma: a marker for poor prognosis. Cancer 83: 34-40, 1998.

17. Cartiana G, Fagotti G, Megathaes A, et al: Glut-1 expression in ovarian carcinoma: association with survival and response to chemotherapy. Cancer 92: 1144-1150, 2001.

18. Younes M, Brown RW, Stephenson M, Gondo M and Cagle PT: Overexpression of Glut1 and Glut3 in stage 1 nonsmall cell lung carcinoma is associated with poor survival. Cancer 80 : 1046-1051, 1997

19. Tohma T, Okazumi S, Makino $\mathrm{H}$, et al: Overexpression of glucose transporter 1 in esophageal squamous cell carcinomas: a marker for poor prognosis. Dis Esophagus 18: 185-189, 2005.

20. Kato H, Takita J, Miyazaki T, et al: Glut-1 glucose transporter expression in esophageal squamous cell carcinoma is associated with tumor aggressiveness. Anticancer Res 22: 2635-2639, 2002.
21. Japanese Society for Esophageal Disease. Guide Lines For The Clinical And Pathologic Studies On Carcinoma Of The Esophagus. 9th edition. Kanehara Shuppan, Tokyo, pp59-79, 1999.

22. Kaplan EL and Meier P: Nonparametric estimation from incomplete observations. J Am Stat Assoc 53: 457-481, 1958.

23. Gehan E: A generalized Wilcoxon test for comparing arbitrarily single-censored samples. Biometrica 52: 203-224, 1965.

24. Cox DR: Regression models and life tables. J R Stat Soc 34: $187-220,1972$.

25. Kawamura T, Kusakabe T, Sugino T, et al: Expression of glucose transporter-1 in human gastric carcinoma: association with tumor aggressiveness, metastasis, and patient survival. Cancer 92: 634-641, 2001.

26. Brophy S, Sheehan KM, McNamara DA, Deasy J, BouchierHayes DJ and Kay EW: GLUT-1 expression and response to chemoradiotherapy in rectal cancer. Int J Cancer 125: 2778-2782, 2009.

27. Hockel M, Schlenger K, Aral B, Mitze M, Schaffer U and Vaupel P: Association between tumor hypoxia and malignant progression in advanced cancer of the uterine cervix. Cancer Res 56: 4509-4515, 1996.

28. Airley RE, Phillips RM, Evans AE, et al: Hypoxia-regulated glucose transporter Glut-1 may influence chemosensitivity to some alkylating agents: Results of EORTC (First Translational Award) study of the relevance of tumour hypoxia to the outcome of chemotherapy in human tumour-derived xenografts. Int J Oncol 26: 1477-1484, 2005.

29. Gray LH, Conger AD, Ebert M, Hornsey S and Scott OC: The concentration of oxygen dissolved in tissues at the time of irradiation as a factor in radiotherapy. Br J Radiol 26: 638-648, 1953.

30. Shetty M, Loeb JN, Vikstrom K and Ismail-Beigi F: Rapid activation of GLUT-1 glucose transporter following inhibition of oxidative phosphorylation in clone 9 cells. J Biol Chem 268 17225-17232, 1993.

31. Warburg O: On the origin of cancer cells. Science 123: 309-314, 1956.

32. Ogawa T, Inugami A, Hatazawa J, et al: Clinical positron emission tomography for brain tumors: comparison of fludeoxyglucose F 18 and L-methyl-(11)C-methionine. Am J Neuroradiol 17: 345-353, 1996.

33. Adams S, Baum R, Schumm-Drager P, Usadel K and Hor G: Limited value of fluorine-18 fluorodeoxyglucose positron emission tomography for the imaging of neuroendocrine tumors Eur J Nucl Med 25: 79-83, 1998.

34. Taylor MD, Smith PW, Brix WK, et al: Correlations between selected tumor markers and fluorodeoxyglucose maximal standardized uptake values in esophageal cancer. Eur J Cardiothorac Surg 35: 699-705, 2009.

35. Westerterp M, Sloof GW, Hoekstra OS, et al: 18FDG uptake in oesophageal adenocarcinoma: linking biology and outcome. J Cancer Res Clin Oncol 134: 227-236, 2008.

36. Kato H, Takita J, Miyazaki T, et al: Correlation of 18-Ffluorodeoxyglucose (FDG) accumulation with glucose transporter (Glut-1) expression in esophageal squamous cell carcinoma. Anticancer Res 23: 3263-3272, 2003. 